\title{
Miíase em felino doméstico (Felis catus Linnaeus 1758) causada por Cochliomyia hominivorax Coquerel, 1858 (Diptera: Calliphoridae) em João Pessoa, Paraíba, Brasil
}

\author{
Aksa Ingrid Vieira Batista 1*, Gabriel Vinicius Carvalho de Lucena 2, Caio Shibuya Pessoa 3, Jessica \\ Azevedo Costeira 3, Roberto Citelli de Farias 3, Josivania Soares Pereira 4 \\ ${ }^{1}$ Departamento de Ciência Animal, Universidade Federal Rural do Semi-Árido, Rio Grande do Norte, RN, Brasil. \\ ${ }^{2}$ Unidade Acadêmica de Medicina Veterinária, Universidade Federal de Campina Grande, Paraíba, PB, Brasil. \\ ${ }^{3}$ Espaço Pet Clínica Veterinária, Paraíba, PB, Brasil. \\ ${ }^{4}$ Departamento de Biociências, Universidade Federal Rural do Semi-Árido, Rio Grande do Norte, RN, Brasil.
}

Research Ethics Committee Approval (if necessary): Not applied.

*Aksa Ingrid Vieira Batista. Rua Francisco Mota Barro, 572 - Mossoró. CEP: 59625-900 - Rio Grande do Norte, RN, Brasil. Telefone: +55 (84) 3317-8247. E-mail: aksaingridmv@gmail.com

Received on: Mar 15, 2021. Accepted on: Mar 26, 2021. Available online: Apr 1, 2021.

\section{Resumo}

Miíase é o nome que se dá a invasão de tecido animal vivo ou morto por larvas de moscas (Diptera). Em animais de companhia, as miíases são consideradas acidentais, principalmente como consequência de feridas abertas. Nas Américas, uma das espécies mais estudadas é a Cochliomyia hominivorax, conhecida como a bicheira do Novo Mundo, devido ao seu grande impacto em animais e humanos. Esse trabalho tem como objetivo relatar a primeira ocorrência de miíase causada por Cochliomyia hominivorax Coquerel 1858 em felino doméstico (Felis catus) no estado da Paraíba, Brasil.

Keywords: Gatos; Moscas; Parasitologia; Ectoparasitismo.

\section{Introdução}

As lesões conhecidas como miíases, correspondem a invasão de tecido animal vivo ou morto por larvas dípteras [1-4]. Esta infecção é considerada uma zoonose de notificação obrigatória pela Organização Internacional de Epizootias (OIE) [5]. Em animais de companhia, essas lesões são consideradas acidentais, principalmente como consequência de feridas abertas, muitas vezes relacionadas às brigas intra $\mathrm{e}$ interespecíficas, principalmente pela competição entre machos. Nestes animais, a negligência dos tutores foi relacionada como um dos principais fatores para a ocorrência desta afecção $[2,5,6]$. 
Miíase em felino doméstico (Felis catus Linnaeus 1758) causada por Cochliomyia hominivorax Coquerel, 1858 (Diptera: Calliphoridae)

\begin{tabular}{|c|c|}
\hline Os felinos domésticos são muito & se não \\
\hline suscetíveis às infestações por & precocemente [1]. \\
\hline $\begin{array}{l}\text { ectoparasitos, apresentando-se muitas } \\
\text { vezes de forma assintomática ou }\end{array}$ & $\begin{array}{c}\mathrm{O} \text { presente estudo tem como } \\
\text { objetivo relatar a primeira ocorrência de }\end{array}$ \\
\hline oligossintomática [1]. Enquanto alguns & miíase causada por Cochliomyia \\
\hline $\begin{array}{l}\text { ectoparasitos, como Ctenocephalides felis } \\
\text { felis Bouché 1835, Felicola subrostratus }\end{array}$ & hominivorax Coquerel $1858 \mathrm{em}$ felino \\
\hline Burmeister 1838, Sarcoptes scabiei De & $\begin{array}{l}\text { doméstico (Felis catus) no estado da } \\
\text { Paraíba, Brasil. }\end{array}$ \\
\hline
\end{tabular}
Geer 1778 e Notoedris cati Hering 1838 são comumente relatados parasitando esses animais, sendo raros os relatos de infestações por larvas de mosca [1].

Nas Américas, uma das espécies mais estudadas é a Cochliomyia hominivorax Coquerel 1858, conhecida como a miíase ou bicheira do Novo Mundo, devido ao seu grande impacto em animais e humanos, afetando as economias de muitos países [7]. Recentemente, C. hominivorax foi incluída na lista de Sistemas Globais de Alerta Precoce para Doenças Principais dos Animais, incluindo Zoonoses, da Organização Mundial da Saúde [8].

As moscas adultas da espécie $C$. hominivorax ovipositam em feridas recentes do hospedeiro, e eclodem nas primeiras 24 horas, penetrando em feridas causadas por acidentes, brigas, picadas de carrapatos ou até mesmo intervenções cirúrgicas [1-2,7-8]. As larvas liberam enzimas proteolíticas que digerem tecidos e produzem lesões extensas [1,8-9].

O diagnóstico da miíase pode ser feito facilmente, com base na identificação das larvas. Infestações massivas são comuns e podem levar à

\section{Relato de caso}

No dia 19 de fevereiro de 2021, deu entrada na Clínica Veterinária Espaço Pet, João Pessoa, Paraíba, Brasil, um felino doméstico, fêmea, sem raça definida, com 1 ano de idade e pesando $4,5 \mathrm{~kg}$.

O animal possuía uma ferida na região perianal, essa se apresentava aberta e ulcerada, além da presença de miíase (Figura 1). $\mathrm{O}$ animal não apresentava quaisquer outros sinais clínicos de doença concomitante. Objetivando a limpeza da área, o animal foi sedado com tiletamina e zolazepam $(0,03 \mathrm{mg} / \mathrm{kg})$.

Então, foi realizada uma tricotomia do local da lesão, as larvas foram retiradas com auxílio de pinças anatômicas, e a ferida foi limpa com clorexidine e solução fisiológica. As larvas dípteras foram eutanasiadas em água morna e depois acondicionadas em álcool $70^{\circ}$ dentro de frascos. Este material foi enviado ao Laboratório de Parasitologia Animal (LPA-UFERSA). Os espécimes foram analisados em estereomicroscopia e microscopia óptica de luz. Para montagem dos mesmos, 
Miíase em felino doméstico (Felis catus Linnaeus 1758) causada por Cochliomyia hominivorax Coquerel, 1858 (Diptera: Calliphoridae)

utilizaram-se métodos adaptados de Almeida et al. [9] de forma que se realizou clareamento em solução de potassa a 30\%; series de hidratações e desidratações, diafanização em fenol. Por fim, estas foram montadas em meio de Hoyer.

Identificou-se 10 larvas do tipo L3 da espécie de Calliphoridae Cochliomyia hominivorax Coquerel, 1858 (Figura 2) a partir de chaves taxonômicas de Laake et al. (1936) [10], Furman e Catts (1982) [11] e Guimarães et al. (1999) [12].

Uma revisão bibliográfica a partir das plataformas Scielo, Pubmed e Google Acadêmico foi realizada nos idiomas inglês e português com o intuito de reunir os relatos de casos de parasitismo por larvas de C. hominivorax Coquerel, 1858 em felinos domésticos no Brasil, onde foram encontrados quatro artigos sobre o assunto (Tabela 1).

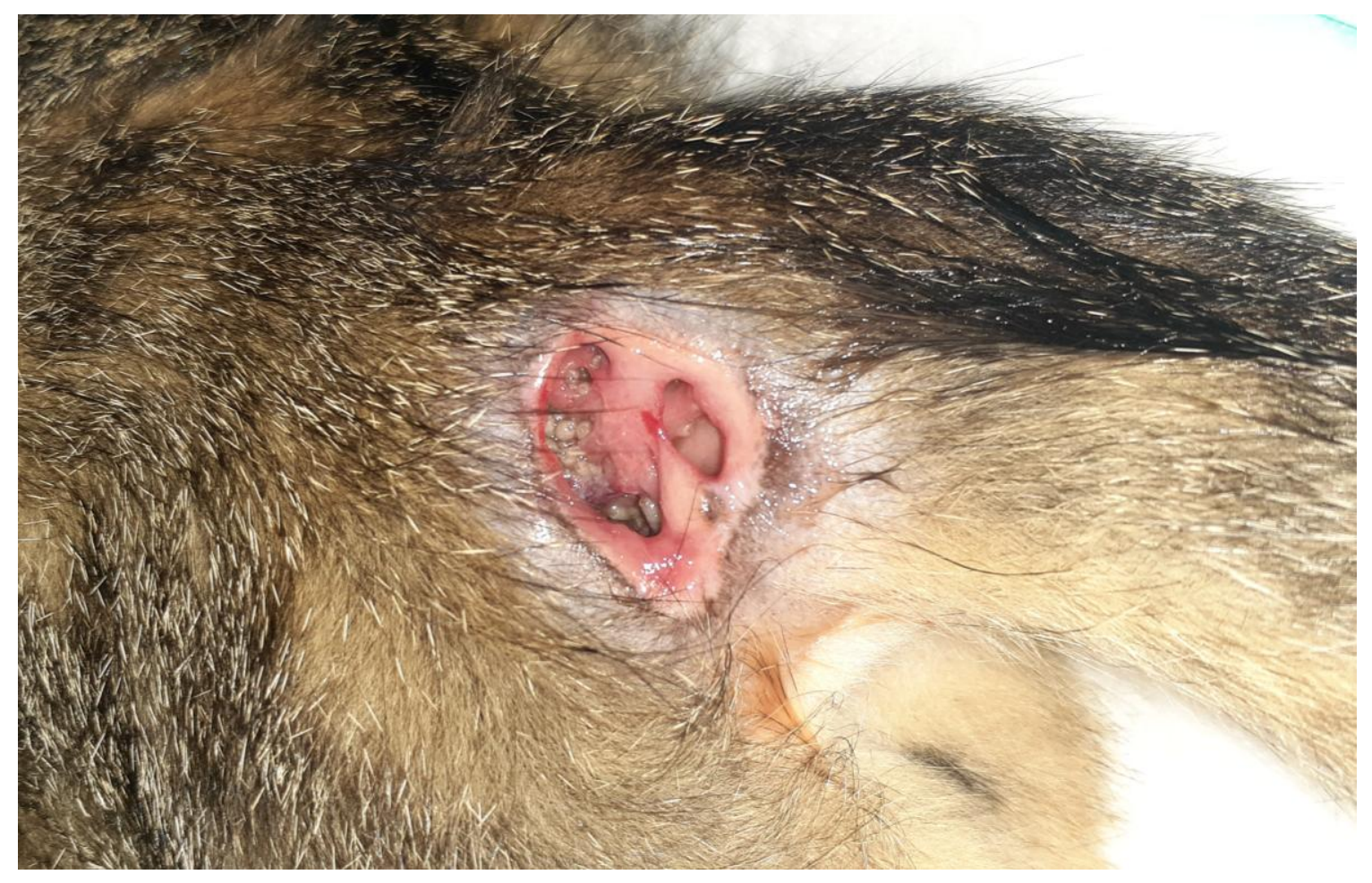

Figura 1. Lesão causada por larvas de Cochliomyia hominivorax Coquerel 1858 em felino doméstico (Felis catus Linnaeus 1758). 
Miíase em felino doméstico (Felis catus Linnaeus 1758) causada por Cochliomyia hominivorax Coquerel, 1858 (Diptera: Calliphoridae)

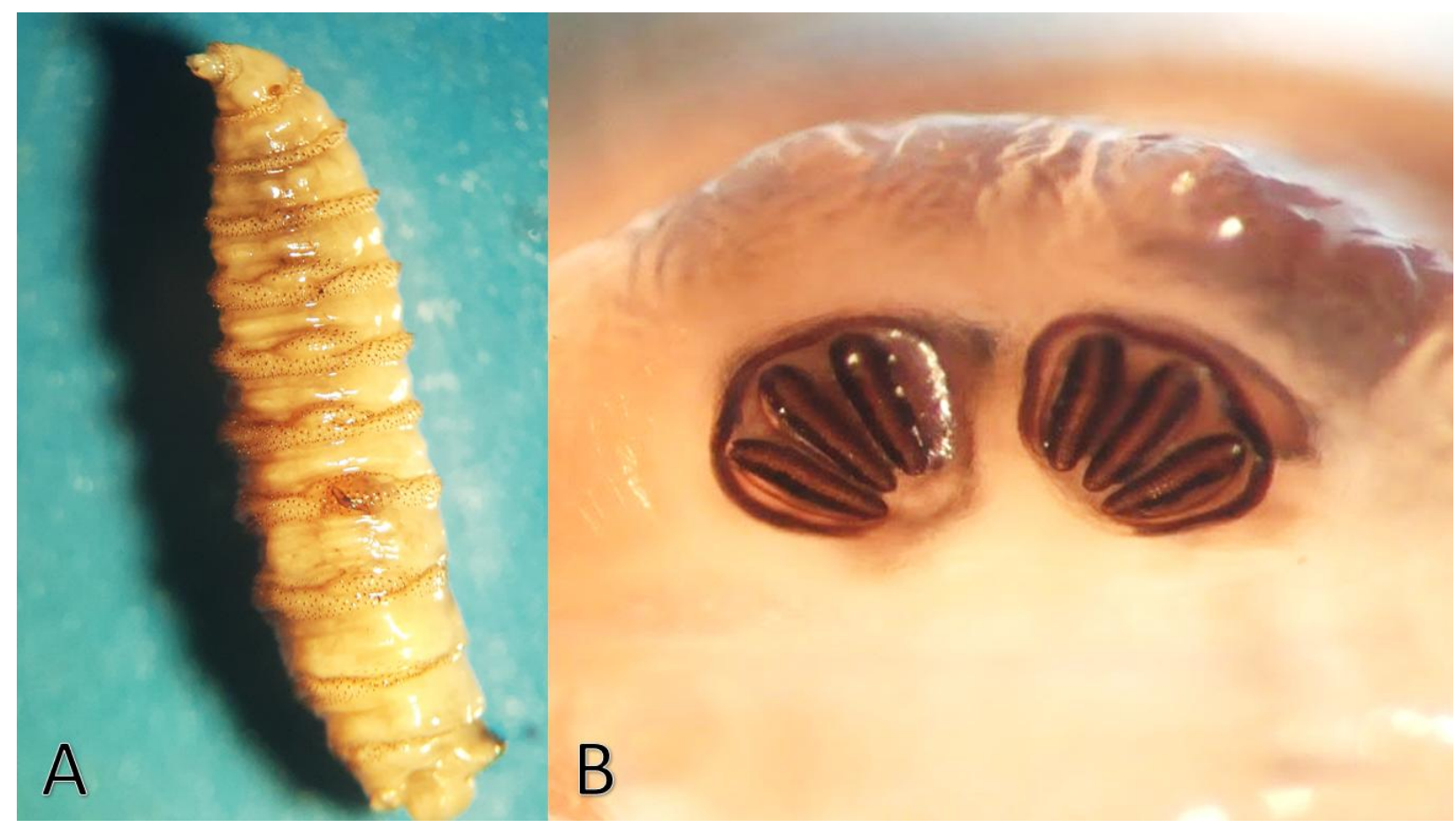

Figura 2. Larva (L3) de Cochliomyia hominivorax Coquerel 1858 em felino doméstico (Felis catus Linnaeus 1758). A. Observação em estereomicroscopia; B. Estigmas respiratórios.

Tabela 1. Casos de parasitismo por larvas de Cochliomyia hominivorax Coquerel, $1858 \mathrm{em}$ felinos domésticos (Felis catus Linnaeus 1758) no Brasil.

\begin{tabular}{lccc}
\hline \multicolumn{1}{c}{ Autor, ano } & $\begin{array}{c}\text { Número de } \\
\text { casos }\end{array}$ & Localização & Referências \\
\hline $\begin{array}{l}\text { Mendes-de- } \\
\text { Almeida et al., 2007 }\end{array}$ & 12 & Rio de Janeiro, RJ, Brasil & {$[1]$} \\
Souza et al., 2009 & 5 & Rio de Janeiro, RJ, Brasil \\
Marotta et al., 2011 & 1 & Rio de Janeiro, RJ, Brasil & {$[8]$} \\
Cansi e Demo, 2011 & 1 & Distrito Federal, Brasil & [5] \\
\hline
\end{tabular}

\section{Discussão e Conclusão}

No Brasil, já foi relatado o parasitismo em gatos apenas pelas larvas de moscas das espécies Dermatobia hominis Linnaeus Jr. 1781, Cochliomyia hominivorax Coquerel 1858 e Lucilia eximia Robineau-Desvoidy 1830 
Miíase em felino doméstico (Felis catus Linnaeus 1758) causada por Cochliomyia hominivorax Coquerel, 1858 (Diptera: Calliphoridae)

[7]. A associação de parasitismo Cochliomyia hominivorax em gatos no Brasil foi relatada apenas nos estados do Rio de Janeiro e Distrito Federal, ainda não havendo relatos na Região Nordeste [1-3,5,8].

Mendes-de-Almeida et al. [1] constatou prevalência entre gatos domésticos infestados por larva de $C$. hominivorax, e a maior incidência das lesões foram na parte frontal do corpo, principalmente face e pescoço, de adultos do sexo masculino, sugerindo que casos de miíases em gatos ocorram em consequência de feridas provocadas por disputas competitivas.

Este relato foge destas características, já que a miíase se encontrava em ferida ulcerada próximo ao ânus de uma fêmea felina. Porém, é válido ressaltar que essas larvas podem estar presentes em basicamente todos os tecidos do corpo, podendo causar perda de estruturas vitais, além de outros sinais que vão variar de onde o ectoparasito está instalado, como: desidratação, caquexia, pirexia, apatia, dispneia, corrimento nasal piossanguinolento, espirros, mucosas hipocoradas, claudicação, entre outros, podendo vir a óbito devido à toxemia, à hemorragia ou a infecções bacterianas secundárias [2].

A ocorrência de C. hominivorax em F. catus é relatada pela primeira vez no Nordeste do Brasil e constitui um novo dado aplicável aos ectoparasitos que ocorrem nessa espécie hospedeira no Brasil. Este encontro colabora para ampliação referente aos dados ecológicos, assim como o bem-estar de gatos-domésticos.

\section{Referências}

[1] Mendes-de-Almeida F, Labarthe N, Guerrero J, Landau-Remy G, Rodrigues DP, Borja GEM, Pereira MJS. Cochliomyia hominivorax myiasis in a colony of stray cats (Felis catus Linnaeus, 1758) in Rio de Janeiro, RJ. Veterinary parasitology. 2007; 146(3): 376-378.

[2] Marotta CR, Scherer PO, Sanavria A. Miíase interna oro-nasal e cutânea por Cochliomyia hominivorax (Coquerel, 1858) em felino (Felis catus)- Relato de caso. Brazilian Journal of Veterinary Medicine. 2011. 33(3): 137-141.

[3] Pezzi M, Bonacci $T$, Leis $M$, Mamolini E, Marchetti MG, Krčmar S, Chicca M, Zingaro CNFD, Faucheux MJ, Scapoli, C. Myiasis in domestic cats: a global review. Parasites \& vectors. 2019; 12(1): 1-14.

[4] Muñoz AAF, Caceres AFB, León JCP. First report of myiasis in dogs caused by Cochliomyia hominivorax (Coquerel 1858) in Colombia. Veterinary Parasitology: Regional Studies and Reports. 2020, 19, 100356.

[5] Cansi MB, Demo C. Ocorrência de miíases em animais de companhia no Distrito Federal, Brasil. Acta Scientiae Veterinariae. 2011; 39(3): 1-5.

[6] Cramer-Ribeiro BC, Sanavria A, Oliveira MQD, Souza FSD, Rocco FDS, 
Miíase em felino doméstico (Felis catus Linnaeus 1758) causada por Cochliomyia hominivorax Coquerel, 1858 (Diptera: Calliphoridae)

Cardoso PG (2002). Inquérito sobre os casos de miíase por Cochliomyia hominivorax em gatos das zonas norte, sul e oeste e do centro do município do Rio de Janeiro no ano 2000. Brazilian Journal of Veterinary Research and Animal Science. 2002; 39(4): 165-170.

[7] Costa-Júnior LM, Chaves DP, Brito DRB, Santos VAFD, Costa-Júnior HN, Barros ATM. A review on the occurrence of Cochliomyia hominivorax (Diptera: Calliphoridae) in Brazil. Revista Brasileira de Parasitologia Veterinária. 2019; 28(4): 548-562.

[8] Souza CP, Verocai GG, Ramadinha RH. Myiasis caused by the New World screwworm fly Cochliomyia hominivorax (Diptera: Calliphoridae) in cats from Brazil: report of five cases. Journal of feline medicine and surgery. 2010; 12(2): 166-168.

[9] Almeida LMD, Ribeiro-Costa CS, Marinoni L. Manual de coleta, conservação, montagem e identificação de insetos. In Manual de coleta, conservação, montagem e identificação de insetos. 2003.

[10] Laake EW, Cushing EC, Parish HE. Biology of the primary screwworm fly, Cochliomyia americana, and a comparison of its stages with those of the Cochliomyia macellaria. 1936.

[11] Furman DP, Catts EP (Eds.). Manual of Medical Entomology, Cambridge University Press, Cambridge. 1982. 72-77.
[12] Guimarães JH, Pavero N. Myiasis in man and animals in the Neotropical region: bibliographic database. São Paulo: Plêiade/FAPESP. 1999.

Conflitos de interesses: Não há nenhum conflito de interesse associado a este artigo.

Agradecimentos: Agradecemos a todos os profissionais e técnicos da Clínica Veterinária Espaço Pet e aos pesquisadores do Laboratório de Parasitologia Animal (LPA-UFERSA).

Financiamento: Nenhum financiamento foi realizado.

Como citar este artigo: Batista AIV, Lucena GVC, Pessoa CS, Costeira JÁ, Farias RC, Pereira JS. Miíase em felino doméstico (Felis catus Linnaeus 1758) causada por Cochliomyia hominivorax Coquerel, 1858 (Diptera: Calliphoridae) em João Pessoa, Paraíba, Brasil. Brazilian Journal of Case Reports. 2021 Jan-Mar;01(2):15-20. 